Do heuristics underpin symptom appraisal for symptoms of cancer?

\title{
Do cognitive heuristics underpin symptom appraisal for symptoms of cancer?: A secondary qualitative analysis across seven cancers
}

\author{
Running title: Do heuristics underpin symptom appraisal for symptoms of \\ cancer?
}

\begin{abstract}
Authors:
Sonja Kummer ${ }^{1}$, Fiona M Walter ${ }^{2,4}$, Joseph Chilcot $^{3}$, Jon Emery ${ }^{4,2}$, Stephen Sutton ${ }^{5}$ and Suzanne E Scott ${ }^{1^{*}}$
\end{abstract}

\begin{abstract}
Affiliations:
${ }^{1}$ Faculty of Dentistry, Oral \& Craniofacial Sciences, King's College London, UK

${ }^{2}$ The Primary Care Unit, Department of Public Health \& Primary Care, University of Cambridge, UK

${ }^{3}$ Health Psychology Section, Psychology Department, Institute of Psychiatry, Psychology and Neuroscience, King's College London

${ }^{4}$ Centre for Cancer Research, Department of General Practice, Faculty of Medicine, Dentistry, and Health Sciences, University of Melbourne, Melbourne, Australia

${ }^{5}$ Behavioural Science Group, Institute of Public Health, University of Cambridge, UK
\end{abstract}

\section{*Corresponding author:}

Dr Suzanne Scott,

Faculty of Dentistry, Oral \& Craniofacial Sciences

This is the author manuscript accepted for publication and has undergone full peer review but has not been through the copyediting, typesetting, pagination and proofreading process, which may lead to differences between this version and the Version of Record. Please cite this article as doi: $10.1002 /$ pon.5049

This article is protected by copyright. All rights reserved. 
King's College London

Floor 18, Guy's Hospital

London SE1 9RT

Email: suzanne.scott@kcl.ac.uk

\section{ABSTRACT}

Objectives: To explore the evidence for cognitive heuristics or 'rules of thumb' used within patients' reports of symptom appraisal and decisions to seek help for symptoms of cancer.

Methods: A secondary analysis of interviews from existing studies that explored symptom appraisal in patients who had sought help for potential symptoms of cancer. Transcripts from $n=50$ in-depth interviews with patients referred with symptoms suspicious of cancer (pancreas, colorectal, oral, lung, melanoma, breast, prostate) were re-analysed using deductive thematic approach underpinned by the heuristics outlined in the Common Sense Model of Illness Self-regulation as set within the Model of Pathways to Treatment.

Results: The most dominant heuristic in patient reports was the Rate of change rule, (i.e. symptoms that are worsening, increasing, or have a sudden onset (rather than improving stable or decreasing in number) are more likely to indicate illness). There was also support for the Duration rule, Pattern rule, Chronology rule, Severity (of interference) rule, Age-illness rule, Novelty rule, Similarity rule, Location rule and Optimistic bias rule. There was a lack of evidence for the Prevalence and Stress-illness rules.

Conclusions: People do appear to use heuristics to guide their appraisal of symptoms and their perceived need for healthcare. Heuristics may be an important aspect underlying symptom misinterpretation, thus making them key targets for interventions. For instance, campaigns could tackle cognitive biases rather than focusing on specific symptom awareness. Myth-busting messages could highlight that intermittent, mild symptoms, and symptoms that are not worsening can be signs of a serious health problem.

This article is protected by copyright. All rights reserved. 
Keywords: cancer, oncology, early diagnosis, help-seeking, heuristics, symptoms; appraisal, delay

This article is protected by copyright. All rights reserved. 


\section{BACKGROUND}

Delays in presentation of symptomatic cancers and problems in the diagnostic pathway can contribute to advanced stage cancer at diagnosis and poor prognosis [1]. If survival rates from cancer are to improve, a greater understanding of the pathways to cancer diagnosis, such as the barriers to seeking help [2] is needed. The Model of Pathways to Treatment [2-3] has been used to explore the pathways to diagnosis for a variety of cancers [4-8], and other conditions [9-10]. The 'appraisal interval', is defined as the time from detection of a bodily change to perceiving a reason to discuss symptoms with a Health Care Practitioner (HCP) [3]. Together with the 'help-seeking interval' it forms the 'patient interval' or 'time to presentation': the time from when a bodily change is first perceived, to when the patient first presents to a HCP.

Due to the non-specific and vague nature of many early cancer symptoms, symptom appraisal explains much of the time to presentation for symptoms of cancer [11-14]. Someone is unlikely to consult a HCP if they believe symptoms are a consequence of a minor ailment that will go away on its own, or one that can be self-managed. Beliefs about symptoms are often more reliable predictors of help-seeking behaviour than symptom type or socio-demographics [15-16]: a person's interpretation of symptoms is key.

What is less clear is what underpins symptom appraisal. How and why does someone decide a bodily change is a sign of indigestion (and take antacids) rather than a sign of a heart attack (and need prompt medical care)? The Common Sense Model of Illness Self-regulation (CSM) is a theoretical model which illustrates how individuals interpret and cope with health threats [17] and could help identify the contributing factors within the appraisal interval. An often overlooked aspect of the CSM is the notion that to help interpret symptoms, individuals rely on heuristics, or so called 'rules of thumb'. Tversky and Kahneman [18] first defined heuristics as logical shortcuts that are used to condense difficult mental operations to simpler cognitive tasks [19]. Leventhal and colleagues $[20,21]$ have identified numerous heuristics that might be used to form a representation of symptoms and decide whether they require medical care (see Supplementary File 1). For instance, according to the Novelty rule, symptoms that are new, different, or incongruent (unexpected) with underlying schema rather than 
familiar, common, or similar to a co-existing chronic illness can be a key motivator to seek help. Another heuristic is the Age-illness rule. According to this heuristic, as individuals age, symptoms will often be attributed to the ageing process rather than illness, and help-seeking will be less likely [22]. Nevertheless, to date there has been a lack of research that has explicitly investigated heuristics that may underlie symptom appraisal in general and cancer symptom appraisal in particular. We do not know if cognitive heuristics underpin symptom appraisal for symptoms of cancer. The aim of this study was to explore the evidence for heuristics used within patients' reports of their decision to seek help for potential symptoms of cancer.

This article is protected by copyright. All rights reserved. 


\section{METHODS}

\section{Study design}

A secondary analysis of existing qualitative data obtained from four studies from the UK and Australia [4-5,8,23-26] between 2003 and 2013 was undertaken, examining symptom appraisal and help-seeking behaviour among people with potential symptoms of cancer relating to seven different sites. These were our own data sets which had previously been analysed using inductive qualitative analysis. All four studies recruited participants in secondary care. Some participants had been recently diagnosed with cancer; others were investigated for cancer and were later diagnosed to have benign disease. Topic guides for the interviews did not specifically ask about heuristics.

\section{Data Sources}

Verbatim transcripts of interviews were selected using the criteria as outlined below. These selection criteria were chosen in order to obtain a wide range of individuals and responses to symptoms. For each of the cancer sites we asked for:

-2-3 participants who had the longest time to presentation (one aged under 60 and one over 60 years)

- 2-3 participants who had the shortest time to presentation (one aged under 60 and one over 60 years)

- 2 participants with time to presentation around the median for that sample (one aged under 60 and one over 60 years).

Extra transcripts were added to achieve similar numbers of men and women and to reach data saturation, giving a total of 50 transcripts. Further details of the data sources can be found in Supplementary File 2. Existing ethical approvals were in place for analysis of the datasets in relation to symptom appraisal and help-seeking behaviours by the same research team ${ }^{1}$. All participants had given written informed consent.

\footnotetext{
1 Guy's Research Ethics Committee 03/03/09[22]; Cambridgeshire 3 Research Ethics Committee 10/H0306/50[4-5,23]; Cambridgeshire 4 Research Ethics Committee 11/EE/0076 [8]; Human Research Ethics Committee of The University of Western Australia (RA/4/1/2242) [24-25].
} 


\section{Data Analysis}

A deductive thematic approach was chosen to actively explore the evidence for heuristics used within patients' reports of their symptom appraisal and decision to seek help for cancer symptoms. Taking a systematic approach, all the contributing factors within the 'appraisal interval' and 'help-seeking interval' as outlined by Scott et al. (2013) in the Model of Pathways to Treatment were coded (see Supplementary File 3 for a detailed categorisation matrix) to ensure all text relevant to the time to presentation was considered. Any text that could not be coded into one of the categories outlined in the categorisation matrix was coded as "other" and was later reexamined and given a new code if required. This paper solely focuses on the heuristics used in the appraisal interval. Clear definitions for each heuristic were developed using the key concepts highlighted in the CSM, and refined using interim coding that ensured clarity and mutual exclusivity (see Supplementary File 1). Interim coding indicated: i) that there were many similarities between the Stereotypes rule and Similarity rule and in turn these were combined. For this analysis, we focused on similarity between oneself and those who develop cancer; ii) symptom interpretation was sometimes guided by the timing of the symptom as opposed to the location (area in the body) of the symptom as stated in the Location rule. Therefore, a separate heuristic (Chronology rule) was generated; iii) refinement of the definitions for Severity rule and Pattern rule was necessary to allow clear differentiation between the two. Pattern rule refers to the nature of symptoms and Severity (of interference) rule refers to the impact of symptoms.

Throughout the analysis process we actively searched for text that was contradictory to each heuristic as well as that which supported it to further demonstrate how respondents may or may not use heuristics to interpret symptoms and decide whether to seek medical care. One transcript from each cancer type was read and independently coded by FMW and SES. This coding was then compared with the main coding conducted by SK. Any discrepancies in coding were discussed until agreement was reached. Data management and coding were facilitated by NVivo software (V.10).

This article is protected by copyright. All rights reserved. 


\section{RESULTS}

\section{Sample characteristics}

Characteristics of the 50 patients are detailed in Supplementary File 4. Twenty-eight participants (57\%) were female. The mean age of participants was 62 years (SD = 12.3).

\section{Evidence of Heuristics}

Figure 1 represents the number of participants coded as using each heuristic. Most participants ( $n=42,84 \%$ ) demonstrated evidence of using at least one heuristic in their accounts of symptom appraisal (range 0 - 7). The average number of heuristics evidenced per participant was 2. This differed across cancer types: pancreatic, colorectal, melanoma, and prostate cancer: average number of heuristics per participant $=3$; lung breast cancer: average number of heuristics per participant $=2$; oral cancer: average number of heuristics per participant $=1$.

Quotes that were contradictory of a heuristic were, in contrast, relatively rare, with only 7 contradictory quotes $(n=4$ for the Rate of Change rule, $n=1$ for the Severity rule, the Novelty rule and the Optimistic Bias rule) across the 50 participants.

Detailed evidence and examples for each heuristic are presented below. Throughout this section quotations are accompanied by information about gender, age, cancer type and time to presentation (TTP).

\section{Rate of Change rule}

Evidence for the Rate of Change rule was found across all seven cancer types. For these individuals, a change in the nature of their symptoms, such as experiencing additional changes or worsening symptoms, altered their symptom appraisal and ultimately provided the motivation to seek help.

"Then it got like a spot come on it so only like in the corner of it and I was like oh that's really new so I should go and get that looked at." [Melanoma, Female, 36 years, Median TTP]

This article is protected by copyright. All rights reserved. 
"It certainly wasn't getting any better, it was getting worse if anything and so I thought it was worthwhile getting it checked out." [Pancreas, Male, 79 years, Median TTP]

Nevertheless, there were four cases where the Rate of Change rule did not appear to affect symptom appraisal. For example, one participant noted that symptoms were worsening; however, help was not sought as she had hoped that the symptoms would get better.
"It just got a bit worse and a bit worse ... and I just compensated and I compensated...then it started getting a bit hard and then I thought oh God this isn't so good, but I thought well you know it still might sort itself out." [Oral, Female, 50 years, Longest TTP]

\section{Duration rule}

Duration of symptoms was a motivator towards initiating help-seeking for all cancer types, except patients with symptoms of breast cancer. Many individuals initially adopted a 'watchful waiting' approach, and decided to monitor their symptoms, often for a prolonged period. On-going bodily changes eventually led individuals to interpret their symptoms as something more serious and to decide that help should be sought.

"After a few days it didn't go down or anything so I thought oh l'd better go and get it checked out." [Melanoma, Female, 54 years, Shortest TTP]

"I've had this wretched cough since around Christmas time, and I am getting a bit worried about it, I really feel it shouldn't go on this long. " [Lung, Female, 74 years, Longest TTP]

\section{Chronology rule}

There was evidence that individuals with symptoms of pancreatic, colorectal, lung and prostate cancer and melanoma used the Chronology rule, whereby explanations given for the cause of symptoms were contextualised as a reaction to specific events. For

This article is protected by copyright. All rights reserved. 
example, individuals with gastro-intestinal symptoms often made a link between their symptoms and the type of food they had recently eaten.

"If I had a mild tummy pain I thought 'oh dear, I shouldn't have had whatever I had to eat last night' [Colorectal, Female, 60 years, Longest TTP]

\section{Pattern rule}

There was evidence for the Pattern rule for all cancer types except those with symptoms of prostate cancer. This rule was particularly evident among those with symptoms of lung cancer where more extreme symptoms triggered perceptions of seriousness and the need for medical help.

"Because every time when I coughed it felt like as if I was ripping my lungs apart, it was really painful." [Lung, Female, 50 years, Shortest TTP]

\section{Severity (of interference) rule}

Evidence for the Severity rule was found across all cancer types, apart from breast cancer. Some participants thought that their symptoms might indicate something serious once symptoms affected their mobility or daily living activities. For example, being unable to stand or get out of bed or having to lie down. In turn, the urgency to seek help immediately was acknowledged.

"But I'd called the paramedics that morning, because by this time I... I was in such agony with my back, I could hardly stand, I couldn't get to the toilet on me own, I couldn't get out of bed on my own." [Lung, Female, 54 years, Shortest TTP]

Also in support of the Severity rule, individuals were often not concerned about their symptoms if they experienced bodily changes that allowed normal functioning to continue. If this was the case, help-seeking was not seen as a necessary course of action.

"And just because of one spot on my leg of course I don't need a doctor because I still I am going to sport centre, I am working, I am doing at home

This article is protected by copyright. All rights reserved. 
everything so I can do it, and yeah, I feel fine." [Melanoma, Female, 40 years, Longest TTP]

On the other hand, contrary to the Severity rule, although one participant's symptom disrupted her normal functioning, she did not seek help because she found a way of adapting to her symptoms.

“Sometimes I couldn't go into lectures until it had gone off. I used to have to lay down on the seat." [Pancreas, Female, 57, Longest TTP]

\section{Novelty rule}

There was some evidence for the Novelty rule from all cancer groups. Individuals often became concerned when they experienced symptoms that were new or different from symptoms experienced in the past, or if symptoms were unexpected.

"I'd never seen anything like that before, and that's what suddenly made me curious and wonder what it was." [Melanoma, Male, 72 years, Longest TTP]

"So I'm thinking, I'm really in trouble now because I never had a lump in my armpit before." [Breast, Female, 56 years, Median TTP]

Furthermore, previous experiences of a condition could dissuade a participant of the potential seriousness of new bodily changes. For instance, a female who presented with melanoma symptoms failed to seek help immediately because the symptoms did not differ from existing symptoms.

"I have a lot of like these spots on my skin so I never paid attention."

[Melanoma, Female, 40 years, Longest TTP]

However, one participant described how her symptoms were not completely new as she had experienced similar symptoms in another location before. This experience helped her to make a link between her previous and new symptoms which in turn promoted help-seeking, rather than diminishing the need to seek help.

This article is protected by copyright. All rights reserved. 
"I'd had ear problems with my ear on the other side and I just thought I really can't afford to have anything with this ear happen as well. So that was another push for me to get something done about it." [Oral, Female, 50 years, Longest TTP]

Age-illness rule

The Age-illness rule was mainly referred to by those with symptoms of prostate and colorectal cancer and melanoma, and less so by those with symptoms of breast and pancreatic cancer. The cause of symptoms was attributed to old age rather than a specific illness.

"You know, you get to my age, you get all sorts of symptoms, but you know, it's age, things don't work as well." [Colorectal, Female, 67 years, Longest $\underline{\text { TTP] }}$

\section{Similarity rule}

The Similarity rule was most commonly evidenced by individuals with melanoma, but there was also evidence among those with symptoms of oral, colorectal, prostate and pancreatic cancer. Reports from individuals with symptoms of breast and lung cancer did not demonstrate use of this heuristic.

When individuals thought that they had a personal predisposition to cancer, for example because of family history or because friends had been diagnosed with cancer, this often guided their symptom appraisal.

"And I went to see my doctor, I was a bit worried then because my boy at 40 he had a melanoma on his arm and at 50 [...] And ah, you know, more or less the reason that I went was because of my boy." [Melanoma, Male, 84 years, Median TTP]

"Well I thought something could be wrong 'cause I [...] I've got five friends, two of them died from the cancer. Ah, and there is three others that I know that have been treated." [Prostate, Male, 82 years, Longest TTP]

This article is protected by copyright. All rights reserved. 


\title{
Location rule
}

There was a little evidence of use of the Location rule, the location of bodily changes guided symptom appraisal and subsequent initiation of help-seeking behaviour for one participant with each cancer type.

\section{"Yeah it's in my lower back and I thought it was part of the injury it was sort right down, just right sort of right down in there." [Prostate, Male, 65 years, Median TTP]}

\section{Optimistic Bias rule}

Evidence in support of use of the Optimistic Bias rule came from participants with symptoms of breast and prostate cancer and melanoma. While bodily changes were often recognised by these participants, help was not sought as they did not consider themselves to be susceptible to illness.

\section{"In my life I don't know, I can count these times when I went to the doctor [...] like I was thinking I am very strong and I am healthy and so I don't need the doctor." [Melanoma, Female, 40 years, Longest TTP]}

\begin{abstract}
However, the Optimistic Bias rule was not confirmed by one participant who explained that, because she had never experienced an illness in the past, the change on her skin might be an indicator that something was wrong, as if she was due to get some form of illness.
\end{abstract}

"I just thought oh, I've never ever had anything wrong, I've not appendix out or anything and I just thought oh maybe this is my thing, um, you know, skin maybe is going to be my problem area." [Melanoma, Female, 54 years, Shortest TTP]

This article is protected by copyright. All rights reserved. 


\section{CONCLUSIONS}

There is a dearth of research into the influence of heuristics on symptom appraisal, yet these findings suggest that heuristics may underlie both symptom interpretation, and, importantly, symptom misinterpretation. This study explored whether accounts of symptom appraisal given by patients with symptoms of a range of cancers demonstrate the use of heuristics as suggested in the CSM [17]. The Rate of Change rule and Duration rule were the most dominant heuristics in patients' reports. There was evidence for use of all the other heuristics apart from the Stress-illness rule and the Prevalence rule although the strength of evidence varied between the heuristics.

Further investigation could characterise which heuristics are most likely to lead to misinterpretation and in turn delays in seeking help, in order to inform the development of tailored interventions. Some heuristics may be more useful than others for interventions. For instance the Rate of Change rule could be used across cancer types whereas the Location rule may be limited to specific cancers. Quantitative measurements are also required for more rigorous hypothesis testing yet tools to measure use of heuristics are currently unavailable. Understanding why there was no evidence for the Stress-illness rule and the Prevalence rule also needs further consideration. For instance, is this because these heuristics are less useful to the interpretation of the studied potential cancer symptoms, or because they are less well articulated? Recent work on changes preceding diagnosis of a brain tumour indicates that attribution to stress is a common response to noticing changes in sleep, mood and cognitive function [27].

We recognise that the sample is too small therefore comparisons between cancer types must be viewed with caution. However, the findings suggest that whilst there are common heuristics across cancer types, there may also be differences in the use of heuristics between individuals with symptoms of different cancers. Different use of heuristics could arise due to variations in the ambiguity of symptoms between these cancer types and differing awareness or recognition of symptoms. For example, recognition of breast cancer warning signs is relatively high compared to other cancers [28], and this may aid symptom appraisal and time to seeking help, and lessen the reliance on heuristics. In contrast, symptoms of melanoma, colorectal and prostate cancer may be less well known or mimic those of minor ailments due to the absence of 
pain or 'alarm' symptoms. In such circumstances individuals may be more likely to use heuristics to understand their symptoms.

\section{Study Limitations}

There are a number of strengths to this study. For instance, data from a diverse group, with seven different cancer types and from two different countries were analysed. However, there are also limitations. For instance, coding the data with the help of a pre-determined categorisation matrix may have biased the exploratory and hypothesisgenerating nature of qualitative analysis. Further, all participants had sought help for their symptoms and as such there is no insight into the symptom appraisal in those who have not sought medical help. Some participants had received a diagnosis of cancer where others had not and this has the potential to affect retrospective accounts.

\section{Theoretical implications}

This is the first study to comprehensively apply the concept of heuristic use to people's accounts of symptom appraisal across a range of cancer types. The process of doing so highlighted that further conceptual work regarding the definitions and wording of heuristics may be required. Some of the existing definitions of the heuristics constructs needed further clarification, or emerged as not mutually exclusive. It also became clear that the definitions of heuristics refer to different aspects of symptom interpretation and help-seeking: while some refer vaguely to interpretation (Location rule), others refer more specifically to causal attribution (e.g. Stress-illness rule), the seriousness of symptoms (e.g. Duration rule), and motivation to seek help (e.g. Novelty rule). Thus people may be using different heuristics for different purposes. Consequently, future work could focus on developing a typology of heuristics to translate the current definitions into more specific criteria for the factors that influence symptom appraisal and help-seeking behaviour. Further research, using content analysis on a larger sample, will also be needed to explore whether heuristics occur in a sequential order and/or whether there are any interactions between heuristics within individuals or the group as a whole and how this impacts symptom appraisal and help-seeking.

This article is protected by copyright. All rights reserved. 


\section{Clinical Implications}

The study provides a novel insight into the elements in symptom appraisal that may in turn trigger or prevent help-seeking behaviour. Heuristics may be an important aspect underlying symptom interpretation and misinterpretation, thus making them key targets for interventions. For instance, campaigns could tackle cognitive biases rather than focusing on specific symptom awareness. Myth-busting messages could highlight that intermittent and mild symptoms and symptoms that are not worsening can be signs of a serious health problem. Such messages would be applicable across cancers and thus a more cost-effective approach. Of course, symptom appraisal is only one part of the help-seeking process. As outlined in the Model of Pathways to Treatment [2-3], contributory factors to the help-seeking interval will also play a role. Thus interventions may need to target heuristics and barriers to seeking help.

\section{ACKNOWLEDGEMENTS}

This work was supported by a King's College London Studentship awarded to Dr Sonja Kummer.

\section{CONFLICT OF INTEREST}

None. 


\section{REFEERNCES}

[1] De Angelis, R., Sant, M., Coleman, M. P., Francisci, S., Baili, P., Pierannunzio, D.,... Group, E.-W. (2014). Cancer survival in Europe 1999-2007 by country and age: results of EUROCARE--5-a population-based study. Lancet Oncol, 15(1), 23-34.

[2] Walter, F., Webster, A., Scott, S. E., \& Emery, J. (2012). The Andersen Model of Total Patient Delay: a systematic review of its application in cancer diagnosis. J Health Serv Res Policy, 17(2), 110-118

[3] Scott, S. E., Walter, F. M., Webster, A., Sutton, S., \& Emery, J. (2013). The model of pathways to treatment: conceptualization and integration with existing theory. Br J Health Psychol, 18(1), 45-65.

[4] Birt, L., Hall, N., Emery, J., Banks, J., Mills, K., Johnson, M., . . W Walter, F. M. (2014). Responding to symptoms suggestive of lung cancer: a qualitative interview study. BMJ Open Respiratory Research, 1(1).

[5] Hall, N., Birt, L., Banks, J., Emery, J., Mills, K., Johnson, M., . . W Walter, F. M. (2015). Symptom appraisal and healthcare-seeking for symptoms suggestive of colorectal cancer: a qualitative study. BMJ Open, 5(10).

[6] Marcu, A., Lyratzopoulos, G., Black, G., Vedsted, P., \& Whitaker, K. L. (2016). Educational differences in likelihood of attributing breast symptoms to cancer: a vignette-based study. Psychooncology, 25(10), 1191-1197.

[7] Moodley, J., Cairncross, L., Naiker, T., \& Momberg, M. (2016). Understanding pathways to breast cancer diagnosis among women in the Western Cape Province, South Africa: a qualitative study. BMJ Open, 6(1).

[8] Walter, F., Birt, L., Cavers, D., Scott, S., Emery, J., Burrows, N., . . Campbell, C. (2014). 'This isn't what mine looked like': a qualitative study of symptom appraisal and help seeking in people recently diagnosed with melanoma. BMJ Open, 4(7).

[9] Liddle, J., Roddy, E., Mallen, C. D., Hider, S. L., Prinjha, S., Ziebland, S., \& Richardson, J. C. (2015). Mapping patients' experiences from initial symptoms to gout diagnosis: a qualitative exploration. BMJ Open, 5(9).

This article is protected by copyright. All rights reserved. 
[10] Usher-Smith, J. A., Thompson, M. J., Zhu, H., Sharp, S. J., \& Walter, F. M. (2015). The pathway to diagnosis of type 1 diabetes in children: a questionnaire study. BMJ Open, 5(3).

[11] Andersen, B., Cacioppo, J., \& Roberts, D. (1995). Delay in seeking a cancer diagnosis: Delay stages and psychophysiological comparison processes. $\mathrm{Br} \mathrm{J}$ Social Psychol 34, 33-52.

[12] Asch, S., Leake, B., Anderson, R., \& Gelberg, L. (1998). Why do symptomatic patients delay obtaining care for tuberculosis? Am J Respir Crit Care Med, 157(4 Pt 1), 1244-1248.

[13] de Nooijer, J., Lechner, L., \& de Vries, H. (2001). A qualitative study on detecting cancer symptoms and seeking medical help; an application of Andersen's model of total patient delay. Patient Educ Couns, 42(2), 145-157.

[14] Molassiotis, A., Wilson, B., Brunton, L., \& Chandler, C. (2010). Mapping patients' experiences from initial change in health to cancer diagnosis: a qualitative exploration of patient and system factors mediating this process. European Journal of Cancer Care, 19(1), 98-109.

[15] Whitaker, K.L, Friedemann Smith, C., Winstanley, K., et al. (2016). What prompts help-seeking for cancer 'alarm' symptoms? A primary care based survey. Br J Cancer, 114, 334-9.

[16] Elliott, A.M., McAteer, A., Hannaford, P.C. (2011). Revisiting the symptom iceberg in today's primary care: results from a UK population survey. BMC Family Practice, 12, 16.

[17] Leventhal, H., Meyer, D., \& Nerenz, D. R. (1980). The common-sense model of illness danger. In S. E. Rachman (Ed.), Medical psychology (Vol. II). New York: Pergamon Press.

[18] Tversky, A., \& Kahneman, D. (1974). Judgment under Uncertainty: Heuristics and Biases. Science, 185(4157), 1124-1131.

[19] Kahneman, D., Slovic, P., \& Tversky, A. (1982). Judgement Under Uncertainty: Heuristics and Biases. Cambridge: Cambridge University Press.

[20] Leventhal, H., Forster, R., \& Leventhal, E. (2007). Self-regulation of health threats, affect and the self: Lessons from older adults. In C. M. Aldwin, C. L.

This article is protected by copyright. All rights reserved. 
Park, \& A. Spiro (Eds.), Handbook of health psychology and aging. New York: Guilford Press.

[21] Leventhal, H., Brisette, I., \& Leventhal, E. A. (2003). The common-sense model of self-regulation of health and illness. In The self-regulation of health and illness behaviour (pp. 41-65). New York: Routledge.

[22] Prohaska, T. R., Keller, M. L., Leventhal, E. A., \& Leventhal, H. (1987). Impact of symptoms and aging attribution on emotions and coping. Health Psychol, 6(6), 495-514.

[23] Scott, S. E., Grunfeld, E. A., Main, J., \& McGurk, M. (2006). Patient delay in oral cancer: a qualitative study of patients' experiences. Psychooncology, 15(6), 474-485.

[24] Banks, J., Walter, F. M., Hall, N., Mills, K., Hamilton, W., \& Turner, K. M. (2014). Decision making and referral from primary care for possible lung and colorectal cancer: a qualitative study of patients' experiences. Br J Gen Pract, 64(629), e775-782.

[25] Emery, J. D., Walter, F. M., Gray, V., Sinclair, C., Howting, D., Bulsara, M., . . . Holman, C. D. (2013). Diagnosing cancer in the bush: a mixed-methods study of symptom appraisal and help-seeking behaviour in people with cancer from rural Western Australia. Fam Pract, 30(3), 294-301.

[26] Emery, J. D., Walter, F. M., Gray, V., Sinclair, C., Howting, D., Bulsara, M., ... Holman, C. D. (2013). Diagnosing cancer in the bush: a mixed methods study of GP and specialist diagnostic intervals in rural Western Australia. Fam Pract, 30(5), 541-550.

[27] Scott, S.E., Penfold, P., Saji, S., Curtis, S., Watts, C., Hamilton, W., Joannides, A., Walter, F.M. (Submitted) 'It was nothing that you would think was anything': Qualitative analysis of appraisal and help seeking for changes preceding brain cancer diagnosis.

[28] Quaife, S., Forbes, L., Ramirez, A., Brain, K., Donnelly, C., \& Simon, A. (2014). Recognition of cancer warning signs and anticipated delay in help-seeking in a population sample of adults in the UK. Br J Cancer, 110(1), 12-18.

This article is protected by copyright. All rights reserved. 
Figure 1. Number of participants appearing to use each heuristic

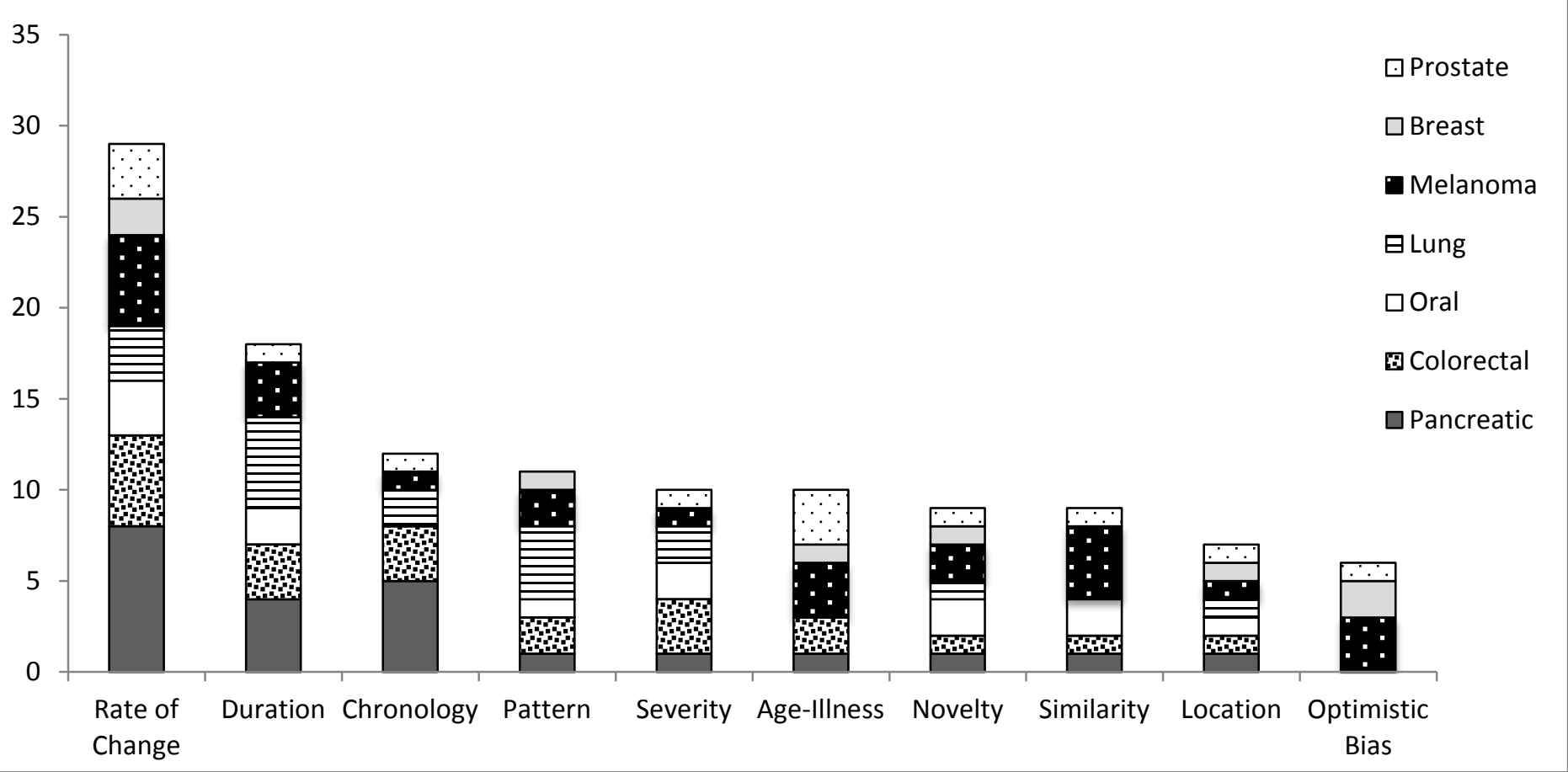

This article is protected by copyright. All rights reserved. 
This article is protected by copyright. All rights reserved. 
Figure 1. Number of participants appearing to use each heuristic

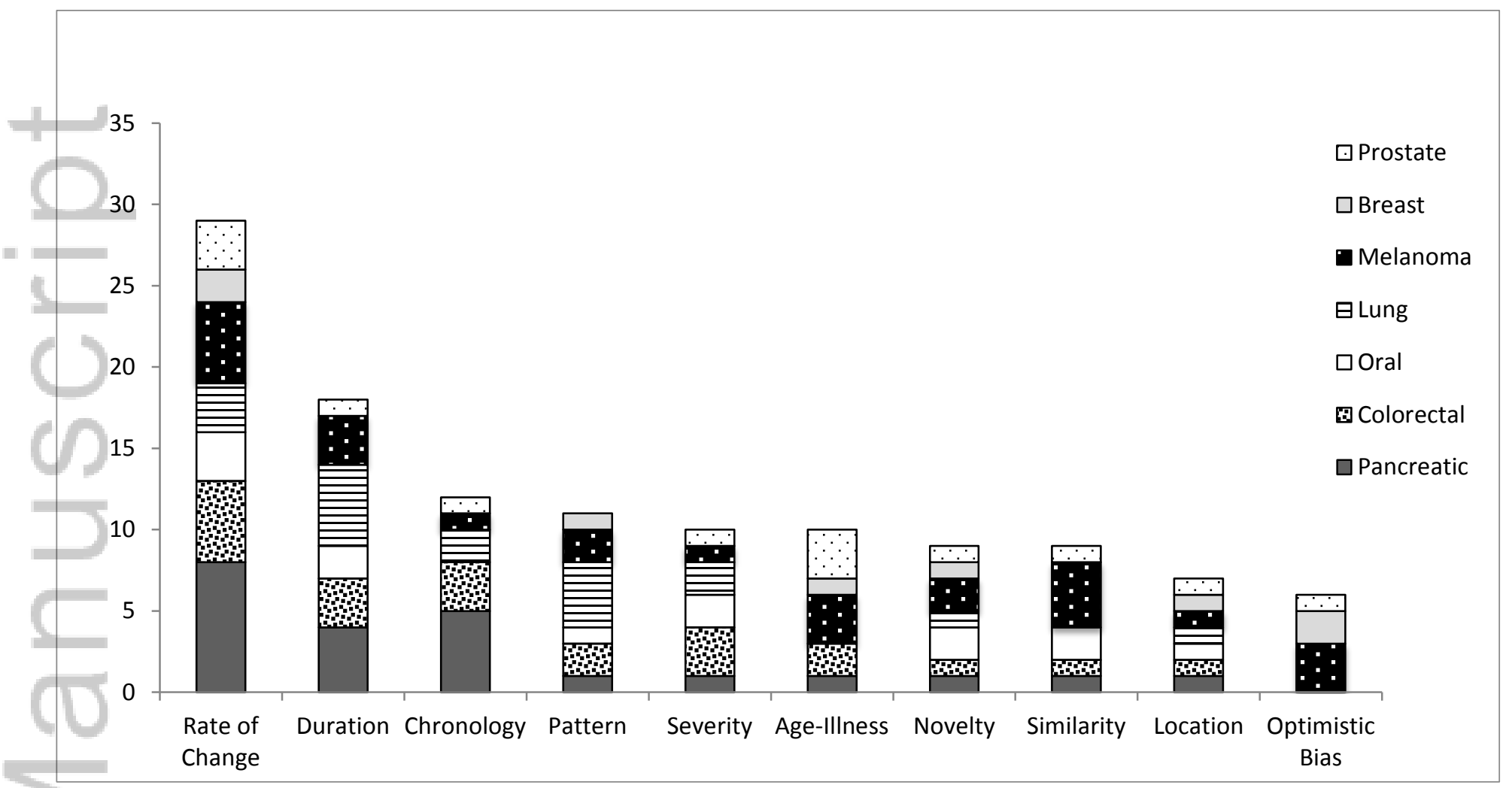

This article is protected by copyright. All rights reserved. 


\section{University Library}

\section{- M M I N E R VA A gateway to Melbourne's research publications}

Minerva Access is the Institutional Repository of The University of Melbourne

Author/s:

Kummer, S;Walter, FM;Chilcot, J;Emery, J;Sutton, S;Scott, SE

Title:

Do cognitive heuristics underpin symptom appraisal for symptoms of cancer?: A secondary qualitative analysis across seven cancers

Date:

2019-05-01

Citation:

Kummer, S., Walter, F. M., Chilcot, J., Emery, J., Sutton, S. \& Scott, S. E. (2019). Do cognitive heuristics underpin symptom appraisal for symptoms of cancer?: A secondary qualitative analysis across seven cancers. PSYCHO-ONCOLOGY, 28 (5), pp.1041-1047. https:// doi.org/10.1002/pon.5049.

Persistent Link:

http://hdl.handle.net/11343/285594 